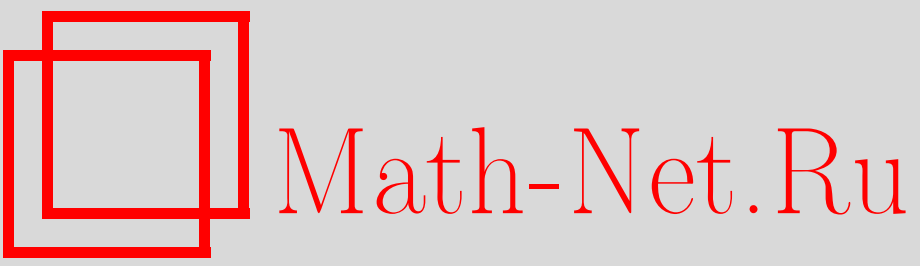

В. М. Гончаренко, О многосолитонных решениях матричного уравнения КдФ, TMФ, 2001, том 126, номер 1, 102-114

DOI: https://doi.org/10.4213/tmf417

Использование Общероссийского математического портала Math-Net.Ru подразумевает, что вы прочитали и согласны с пользовательским соглашением

http://www . mathnet.ru/rus/agreement

Параметры загрузки:

IP: 52.23 .180 .231

26 апреля 2023 г., 15:57:39 


\section{О МНОГОСОЛИТОННЫХ РЕШЕНИЯХ МАТРИЧНОГО УРАВНЕНИЯ КДФ}

Рассматриваются многосолитонные решения матричного уравнения $\mathrm{Kд} \Phi$. Получены формулы для сдвига фаз и изменения амплитуд при взаимодействии двух солитонов, и показано, что многочастичный эффект при многосолитонном взаимодействии отсутствует. Найдены условия, гарантирующие симметричность соответствующих решений матричного уравнения КдФ, полученных матричными преобразованиями Дарбу оператора Шредингера с нулевым потенциалом.

Одним из самых замечательных свойств нелинейных эволюционных уравнений, интегрируемых методом обратной задачи, является существование многосолитонных решений. В частности, $N$-солитонные решения уравнения КдФ могут быть получены с помошью уравнения Гельфанда-Левитана-Марченко для оператора Шредингера (см. [1], [2]) при условии, что коэффициент отражения тождественно равен нулю, или с помощью преобразований Дарбу (см., например, [3]). Замечательной особенностью этих решений является то, что они могут быть интерпретированы как решения, описывающие взаимодействие $N$ солитонов, которые после взаимодействия не меняют своей формы, а лишь претерпевают некоторый сдвиг фаз. Точнее, если $0<\lambda_{1}<\cdots<\lambda_{N}$ - некоторые положительные числа, определяющие скорость $4 \lambda_{k}^{2} k$-го солитона, и $\phi_{k}^{ \pm}-$его фазы при $t \rightarrow \pm \infty$, то сдвиг фаз $\Delta_{k} k$-го солитона задается формулой

$$
\Delta_{k}=\phi_{k}^{+}-\phi_{k}^{-}=\frac{1}{2 \lambda_{k}} \sum_{n=1}^{k-1} \ln \left(\frac{\lambda_{k}+\lambda_{n}}{\lambda_{k}-\lambda_{n}}\right)^{2}-\frac{1}{2 \lambda_{k}} \sum_{n=k+1}^{N} \ln \left(\frac{\lambda_{k}-\lambda_{n}}{\lambda_{k}+\lambda_{n}}\right)^{2} .
$$

В этой работе исследуется взаимодействие солитонов матричного уравнения КдФ

$$
U_{t}=3 U U_{x}+3 U_{x} U-U_{x x x}
$$

где $U(x, t)-(d \times d)$-матрица, не обязательно симметричная. Это уравнение было введено Лаксом [4]. Вадати и Камиджо [5], а затем Калоджеро и Дегасперис [6] рассмотрели широкий класс интегрируемых эволюционных уравнений, связанных с матричным оператором Шредингера

$$
L=-D^{2}+U(x), \quad D=\frac{d}{d x}, \quad x \in \mathbb{R}
$$

\footnotetext{
*Финансовая академия, Москва, Россия. E-mail: mike-gonchar@mtu-net.ru
} 
с эрмитовым потенциалом $U^{*}=U$. Дегасперис в работе [7] детально исследовал взаимодействие солитонов уравнения бумерона. Как это ни странно, подобное исследование солитонных решений матричного уравнения КдФ до сих пор не было проделано, хотя никаких принципиальных трудностей это не вызывает. Мы будем опираться на результаты Мартинеса Алонсо и Олмедиллы [8], [9], которые рассматривали прямую и обратную задачи для оператора (2) с обшим (неэрмитовым) потенциалом.

В первом разделе, используя понятие квазидетерминанта, введенное Гельфандом и Ретахом [10], мы получим обшую формулу для многосолитонного решения уравнения (1) в терминах спектральных данных соответствующего оператора Шредингера.

Во втором разделе мы построим решение уравнения $\mathrm{K} д \Phi$, представляюшее собой семейство солитонов с одинаковыми скоростями, но различными фазами (параллельные солитоны). Затем мы выведем формулы для сдвига фаз солитонов при их взаимодействии для двухсолитонного решения матричного уравнения $\mathrm{Kд} \Phi$, из которых видно, что в матричном случае картина взаимодействия гораздо богаче, чем в скалярном. В частности, меняются как фазы, так и (матричные) амплитуды солитонов; при некоторых начальных амплитудах, заданных несимметричными матрицами, медленный солитон сдвигается вперед, а быстрый - назад, в противоположность тому, что имеет место в скалярном случае (см. [2]).

В третьем разделе мы изучаем связь между двумя подходами к построению многосолитонных решений матричного уравнения $\mathrm{K} д \Phi$ - преобразованиями Дарбу и уравнениями Гельфанда-Левитана-Марченко.

Часть результатов этой статьи была анонсирована в [11], где также обсуждалась связь солитонных матричных потенциалов с безмонодромными (см. также [12]).

\section{1. МНОГОСОЛИТОННЫЕ РЕШЕНИЯ МАТРИЧНОГО УРАВНЕНИЯ КДФ}

Напомним некоторые факты из теории обратного преобразования рассеяния для матричного оператора Шредингера $(2)$ с потенциалом $U(x)$, заданным матричной $(d \times d)$ функцией, вообше говоря, несимметричной и удовлетворяющей условию

$$
\int_{-\infty}^{+\infty}\left(1+x^{2}\right)|U(x)| d x<\infty
$$

где $|X|=\max _{j} \sum_{k}\left|X_{j k}\right|$ для некоторой матрицы $X$. В дальнейшем мы следуем работам [8], [9]. Рассмотрим соответствующее уравнение Шредингера

$$
L \Psi=k^{2} \Psi
$$

где $\Psi=\Psi(x, k)$ - также $(d \times d)$-матричнозначная функция. Определим решения Йоста $F(x, k)$ и $G(x, k) \quad(k \in \mathbf{R})$ уравнения $(4)$, удовлетворяющие условиям

$$
\begin{aligned}
& F(x, k)=e^{i k x}(I+o(1)), \quad x \rightarrow+\infty, \\
& G(x, k)=e^{-i k x}(I+o(1)), \quad x \rightarrow-\infty .
\end{aligned}
$$


Так как $F(x,-k)$ и $G(x,-k)$ - также решения $(4)$, то имеют место соотношения

$$
\begin{aligned}
& G(x, k)=F(x,-k) A(k)+F(x, k) B(k), \\
& F(x, k)=G(x,-k) C(k)+F(x, k) D(k),
\end{aligned}
$$

где $A(k), B(k), C(k), D(k)$ - некоторые $(d \times d)$-матрицы. Матриша $A(k)$ аналитична в области $\operatorname{Im} k>0$ и непрерывна вплоть до вешественной оси. Решения $k_{j}$ уравнения

$$
\operatorname{det} A(k)=0
$$

в верхней полуплоскости соответствуют дискретному спектру оператора $L$ с векторными собственными функциями из $L_{2}(-\infty, \infty)$. Мы будем предполагать, что полюсы $A^{-1}(k)$ простые и $A(k)$ - невырожденная матрица на действительной оси.

ЗАмЕчАниЕ. Если $U(x)$ - эрмитова матрица, удовлетворяющая условию (3), то последние два условия выполнены автоматически (см. [5]). Если же матрица $U(x)$ неэрмитова, то эти предположения существенны, так как $A^{-1}(k)$ может иметь полюсы высшего порядка (см. [9], где исследован случай полюса второго порядка).

Можно также доказать, что число полюсов матрицы $A^{-1}(k)$ конечно (см. [9]).

Пусть теперь $N_{j}=\operatorname{Res}_{k=k_{j}} A^{-1}(k), j=1, \ldots, n$. Тогда сушествуют матрицы $R_{j}$ такие, что

$$
G\left(x, k_{j}\right) N_{j}=i F\left(x, k_{j}\right) R_{j} .
$$

Матричный аналог коэффициента отражения определяется формулой

$$
R(k)=B(k) A^{-1}(k) .
$$

Множество $\left\{k_{j}, R_{j}, R(k)\right\}$ называется данными рассеяния матричного оператора Шредингера. Для решения обратной задачи восстановления оператора $L$ по данным рассеяния положим

$$
H(x)=\sum_{j=1}^{n} R_{j} e^{i k_{j} x}+\frac{1}{2 \pi} \int_{x}^{+\infty} d k R(k) e^{i k x} .
$$

Потенциал оператора $L$ находится по формуле

$$
U(x)=-2 K^{\prime}(x)
$$

где $K(x)=K(x, x)$, а $K(x, y)$ - решение матричного уравнения Гельфанда-ЛевитанаМарченко (см. [9])

$$
K(x, y)+H(x+y)+\int_{x}^{+\infty} K(x, z) H(y+z) d z=0
$$


Матричное уравнение КдФ допускает представление Лакса [4], [5] $L_{t}=[L, B]$, где $L=-D^{2}+U(x, t)$ и $B=-4 D^{3}+3(U(x, t) D+D U(x, t))$. Так же как и в скалярном случае, легко проверить [6], что если $U(x, t)$ - решение уравнения (1), то

$$
\begin{gathered}
A(k, t)=A(k, 0), \quad R_{j}(t)=R_{j}(0) e^{8 i k_{j}^{3} t}, \quad j=1, \ldots, n, \\
R(k, t)=R(k, 0) e^{8 i k^{3} t} .
\end{gathered}
$$

Рассмотрим теперь случай безотражательных потенциалов: $R(k) \equiv 0$. В этом случае уравнения Гельфанда-Левитана-Марченко решаются явно. Предположим, что все $k_{j}: A\left(k_{j}\right)=0$ расположены на мнимой оси, так что $k_{j}=i \lambda_{j}, 0<\lambda_{1}<\cdots<\lambda_{n}$. Тогда уравнение (8) можно переписать в виде

$$
K(x, y)+\sum_{j=1}^{n} R_{j} e^{-\lambda_{j}(x+y)}+\sum_{j=1}^{n} \int_{x}^{+\infty} K(x, z) R_{j} e^{-\lambda_{j}(y+z)} d z=0 .
$$

Предполагая, что $K(x, y)$ имеет вид

$$
K(x, y)=\sum_{j=1}^{n} K_{j}(x) e^{-\lambda_{j} y},
$$

интегрируя и разделяя слагаемые с $e^{-\lambda_{j} y}, j=1, \ldots, n$, мы получаем следуюшую систему линейных уравнений:

$$
\sum_{j=1}^{n} K_{j} \mathcal{A}_{i j}=-R_{j} e^{-\lambda_{j} x}, \quad \mathcal{A}_{i j}=\delta_{i j} I+\frac{R_{j}}{\lambda_{i}+\lambda_{j}} e^{-\left(\lambda_{i}+\lambda_{j}\right) x}, \quad i, j=1, \ldots, n .
$$

Для того чтобы выписать явную формулу для $K(x)$, мы используем понятие квазидетер.минанта, введенное Гельфандом и Ретахом [10].

Пусть $R=\operatorname{Mat}_{d}(\mathbf{C})$ обозначает алгебру $(d \times d)$-матриц и $X-(n \times n)$-матрица над $R$. Для любых $1 \leqslant i, j \leqslant n$ обозначим через $r_{i}(X) i$-ю строку и через $c_{j}(X)-j$-й столбец матрицы $X$. Пусть $X^{i j}$ - подматрица $X$, полученная удалением $i$-й строки и $j$-го столбца $X$. Для вектора-строки $r$ пусть $r^{(j)}$ обозначает $r$ без $j$-го элемента. Для вектора-столбца $c$ пусть $c^{(i)}-c$ без $i$-го элемента. Тогда по определению квазидетерминант

$$
|X|_{i j}=x_{i j}-r_{i}(X)^{(j)}\left(X^{i j}\right)^{-1} c_{j}(X)^{(i)},
$$

где $x_{i j}-i j$-й элемент матрицы $X$. Легко проверить, что если $d=1$, то

$$
|X|_{i j}=(-1)^{(i+j)} \frac{\operatorname{det} X}{\operatorname{det} X^{i j}} .
$$

Обозначим через $\mathcal{A}(n \times n)$-матрицу, $i j$-м элементом которой является $(d \times d)$-матрица $\mathcal{A}_{i j}$. Тогда легко проверить, что

$$
K(x)=\sum_{j=1}^{n}\left|\mathcal{A}^{(j)}\right|_{j j}|\mathcal{A}|_{j j}^{-1},
$$

где $\mathcal{A}^{(j)}$ получается из $\mathcal{A}$ дифференцированием $j$-й строки, а $|\mathcal{A}|_{j j}-j j$-й квазидетерминант матрицы $\mathcal{A}$.

Таким образом, мы приходим к следуюшей теореме (ср. [13]). 
ТеОРема 1. Матричное уравнение КдФ обладает многосолитонными решения.мน

$$
U(x, t)=-2\left(\sum_{j=1}^{n}\left|\mathcal{A}^{(j)}\right|_{j j}|\mathcal{A}|_{j j}^{-1}\right)^{\prime},
$$

где әлементы $\mathcal{A}_{i j}$ матрици $\mathcal{A}$ определяются формулами $(10)$ с $R_{j}(t)=R_{j}(0) e^{8 \lambda_{j}^{3} t}$, a $\left(\lambda_{j}, R_{j}(0)\right)$ - данные рассеяния безотражсательного потенциала $U(x, 0)$.

\section{2. ПРИМЕРЫ МНОГОСОЛИТОННЫХ РЕШЕНИЙ МАТРИЧНОГО УРАВНЕНИЯ КДФ. ВЗАИМОДЕЙСТВИЕ СОЛИТОНОВ}

Далее мы приведем примеры одно- и двухсолитонных решений уравнения КдФ и исследуем взаимодействие его солитонов.

ПримеР 1. Односолитонные решения матричного уравнения КдФ.

Односолитонные решения уравнения (1) соответствуют оператору Шредингера с одним дискретным собственным значением $\lambda$. Предположим сначала, что соответствующая спектральная матрица имеет вид $R=r P$, где $P=P^{2}$-проектор. С использованием выражений $(9)$ уравнение (10) (для $n=1$ ) может быть переписано в форме

$$
K(x, x)\left(I e^{2 \lambda \theta}+\frac{R}{2 \lambda}\right)=-R,
$$

где $\theta=x-4 \lambda^{2} t$. Очевидно,

$$
K(x, x)=-R\left(I e^{2 \lambda \theta}+\frac{R}{2 \lambda}\right)^{-1}=\frac{2 \lambda R}{2 \lambda e^{2 \lambda \theta}+r}
$$

и

$$
U(x, t)=-2 K^{\prime}(x, x)=-2 \lambda^{2} P \operatorname{sech}^{2} \lambda(\theta-\phi), \quad \phi=\frac{1}{2 \lambda} \ln \frac{r}{2 \lambda} .
$$

Мы называем $2 \lambda^{2} P=2 \lambda^{2} R / \operatorname{tr} R$ матричной амплитудой солитона и $\phi$ - его фазой. Проектор $P$ иногда называется поляризацией солитона.

Если

$$
R=\sum_{k=1}^{n} r^{(k)} P^{(k)},
$$

где $P^{(k)}$ - проекторы на соответствуюшие собственные подпространства такие, что $P^{(i)} P^{(j)}=\delta_{i j}$, то

$$
U(x, t)=-2 \lambda^{2} \sum_{k=1}^{n} P^{(k)} \operatorname{sech}^{2} \lambda\left(\theta-\phi_{k}\right), \quad \phi_{k}=\frac{1}{2 \lambda} \ln \frac{r^{(k)}}{2 \lambda} .
$$

Таким образом, матричное уравнение КдФ имеет решение, представляющее собой семейство параллельных солитонов, бегуших с одинаковыми скоростями, но имеюших разные фазы. Это имеет место лишь в матричном случае $(d>1)$ и связано с наличием у соответствуюшего матричного оператора Шредингера кратных собственных значений, когда одному дискретному уровню соответствуют несколько линейно независимых собственных функций, квадратично интегрируемых на прямой. 
ПримеР 2. Двухсолитонное решение матричного уравнения КдФ.

Соответствуюший оператор Шредингера имеет два дискретных уровня: $-\lambda_{1}^{2}$ и $-\lambda_{2}^{2}$. Будем предполагать, что спектральные матрицы имеют вид

$$
R_{j}=r_{j} P_{j}, \quad j=1,2,
$$

где

$$
P_{j}=\frac{p_{j} \otimes q_{j}}{\left(p_{j}, q_{j}\right)}
$$

a $d$-векторы $p_{j}, q_{j}$ удовлетворяют условию $\left(p_{j}, q_{j}\right) \neq 0$. Вводя матрицы $L_{j}=K_{j} e^{-\lambda_{j} x}$ такие, что

$$
K(x, x)=L_{1}(x)+L_{2}(x)
$$

и переменные $\theta_{j}=x-4 \lambda_{j}^{2} t$, мы получаем из формул (10), что $L_{j}$ удовлетворяют системе

$$
\begin{aligned}
& L_{1} e^{2 \lambda_{1} \theta_{1}}+L_{1} \frac{R_{1}}{2 \lambda_{1}}+L_{2} \frac{R_{1}}{\lambda_{1}+\lambda_{2}}=-R_{1}, \\
& L_{2} e^{2 \lambda_{2} \theta_{2}}+L_{1} \frac{R_{2}}{\lambda_{1}+\lambda_{2}}+L_{2} \frac{R_{2}}{2 \lambda_{2}}=-R_{2} .
\end{aligned}
$$

После несложных преобразований получаем

$$
\begin{aligned}
& L_{1}=\frac{\lambda_{1}+\lambda_{2}}{g}\left(R_{2}-\left(\lambda_{1}+\lambda_{2}\right) g_{2} I\right) R_{1}, \\
& L_{2}=\frac{\lambda_{1}+\lambda_{2}}{g}\left(R_{1}-\left(\lambda_{1}+\lambda_{2}\right) g_{1} I\right) R_{2},
\end{aligned}
$$

где

$$
g_{j}=e^{2 \lambda_{j} \theta_{j}}+\frac{r_{j}}{2 \lambda_{j}} \quad \text { для } j=1,2
$$

$$
g=\left(\lambda_{1}+\lambda_{2}\right)^{2} g_{1} g_{2}-\left(p_{1}, q_{2}\right)\left(p_{2}, q_{1}\right),
$$

откуда имеем

$$
K(x, x)=L_{1}+L_{2}=\frac{\left(\lambda_{1}+\lambda_{2}\right)}{g}\left(\left\{R_{1}, R_{2}\right\}-\left(\lambda_{1}+\lambda_{2}\right)\left(g_{2} R_{1}-g_{1} R_{2}\right)\right),
$$

где $\left\{R_{1}, R_{2}\right\}=R_{1} R_{2}+R_{2} R_{1}$ и

$$
U(x, t)=-2 K^{\prime}(x, x)
$$

Важно выделить те начальные данные, при которых солитонное решение КдФ регулярно. Анализируя формулу (14) для $g$, легко получить следующее 
ПРЕДЛОЖЕНИЕ 1. Потенциал (15) регулярен, если $r_{1}>0, r_{2}>0 u \alpha>0$, əде

$$
\alpha=1-\frac{4 \lambda_{1} \lambda_{2}\left(p_{1}, q_{2}\right)\left(q_{1}, p_{2}\right)}{r_{1} r_{2}\left(\lambda_{1}+\lambda_{2}\right)^{2}} .
$$

Исследуем поведение $U(x, t)$ при $|t| \rightarrow \infty$. В окрестности прямой $\theta_{1}=x-4 \lambda_{1}^{2} t=0$ :

$$
\begin{aligned}
& U(x, t) \sim-2 \lambda_{1}^{2} P_{1} \operatorname{sech}^{2} \lambda_{1}\left(\theta_{1}-\phi_{1}^{-}\right), \quad t \rightarrow-\infty, \\
& U(x, t) \sim-2 \lambda_{1}^{2} \widehat{P}_{1} \operatorname{sech}^{2} \lambda_{1}\left(\theta_{1}-\phi_{1}^{+}\right), \quad t \rightarrow+\infty,
\end{aligned}
$$

где

$$
\begin{gathered}
\widehat{P}_{1}=-\frac{\hat{p}_{1} \otimes \hat{q}_{1}}{\left(\hat{p}_{1}, \hat{q}_{1}\right)} \\
\hat{p}_{1}=r_{2} p_{1}-\frac{2 \lambda_{2}}{\left(\lambda_{1}+\lambda_{2}\right)}\left(p_{1}, q_{2}\right) p_{2}, \quad \hat{q}_{1}=r_{2} q_{1}-\frac{2 \lambda_{2}}{\left(\lambda_{1}+\lambda_{2}\right)}\left(p_{2}, q_{1}\right) q_{2}, \\
\phi_{1}^{-}=\frac{1}{2 \lambda_{1}} \ln \frac{r_{1}}{2 \lambda_{1}}, \quad \phi_{1}^{+}=\frac{1}{2 \lambda_{1}} \ln \frac{\left(\hat{p}_{1}, \hat{q}_{1}\right)}{2 \lambda_{1} r_{2}^{2}} .
\end{gathered}
$$

В окрестности прямой $\theta_{2}=x-4 \lambda_{2}^{2} t=0$ :

$$
\begin{aligned}
& U(x, t) \sim-2 \lambda_{2}^{2} \widehat{P}_{2} \operatorname{sech}^{2} \lambda_{2}\left(\theta_{2}-\phi_{2}^{-}\right), \quad t \rightarrow-\infty, \\
& U(x, t) \sim-2 \lambda_{2}^{2} P_{2} \operatorname{sech}^{2} \lambda_{2}\left(\theta_{2}-\phi_{2}^{+}\right), \quad t \rightarrow+\infty,
\end{aligned}
$$

где

$$
\begin{gathered}
\widehat{P}_{2}=\frac{\hat{p}_{2} \otimes \hat{q}_{2}}{\left(\hat{p}_{2}, \hat{q}_{2}\right)}, \\
\hat{p}_{2}=r_{1} p_{2}-\frac{2 \lambda_{1}}{\left(\lambda_{1}+\lambda_{2}\right)}\left(p_{2}, q_{1}\right) p_{1}, \quad \hat{q}_{2}=r_{1} q_{2}-\frac{2 \lambda_{1}}{\left(\lambda_{1}+\lambda_{2}\right)}\left(p_{1}, q_{2}\right) q_{1}, \\
\phi_{2}^{-}=\frac{1}{2 \lambda_{2}} \ln \frac{\left(\hat{p}_{2}, \hat{q}_{2}\right)}{2 \lambda_{2} r_{1}^{2}}, \quad \phi_{2}^{+}=\frac{1}{2 \lambda_{2}} \ln \frac{r_{2}}{2 \lambda_{2}} .
\end{gathered}
$$

Легко также проверить, что в окрестности любой другой прямой $\theta=x-4 \lambda^{2} t, \lambda>0$, $\lambda \neq \lambda_{1}, \lambda \neq \lambda_{2}$ на плоскости $(x, t)$

$$
U(x, t) \rightarrow 0, \text { если }|t| \rightarrow \infty .
$$

Таким образом, мы получаем, что $U(x, t)$ распадается в сумму двух солитонов при $|t| \rightarrow \infty$, причем $j$-й солитон движется со скоростью $4 \lambda_{j}^{2}$. Сдвиги фаз солитонов $\Delta_{j}=$ $\phi_{j}^{+}-\phi_{j}^{-}$определяются формулами

$$
\Delta_{1}=\frac{1}{2 \lambda_{1}} \ln \alpha, \quad \Delta_{2}=-\frac{1}{2 \lambda_{2}} \ln \alpha,
$$


где $\alpha$ задается выражением (16). Из последних формул легко видеть, что $\Delta_{1}\left(\Delta_{2}\right)$ может быть как положительным (отрицательным), так и отрицательным (положительным), что означает, что солитоны могут взаимодействовать различными способами: медленный солитон может сдвигаться назад, а быстрый - вперед, как это было в скалярном случае [2], и наоборот, медленный солитон может сдвигаться вперед, а быстрый - назад.

Другой замечательной особенностью взаимодействия матричных солитонов является изменение их амплитуд вследствие взаимодействия (см. [6]). При изменении $t$ от $-\infty$ до $+\infty$ амплитуда первого солитона меняется от значения $2 \lambda_{1}^{2} P_{1}$ до $2 \lambda_{1}^{2} \widehat{P}_{1}$, а второго - от $2 \lambda_{2}^{2} \hat{P}_{2}$ до $2 \lambda_{2}^{2} P_{2}$. Если $\left(p_{1}, q_{2}\right)=0 \quad\left(P_{2} P_{1}=0\right)$ или $\left(p_{2}, q_{1}\right)=0\left(P_{1} P_{2}=0\right)$, то фазы солитонов не меняются и взаимодействие проявляется лишш как изменение соответствующих амплитуд. Если оба скалярных произведения равны нулю, то солитоны проходят друг через друга без изменений, т.е. они не взаимодействуют (в этом случае $\left.P_{1} P_{2}=P_{2} P_{1}=0\right)$, что соответствует диагонализуемому потенциалу $U(x, t)$ со скалярными 1-солитонами на диагоналях.

Предположим теперь, что спектральные матрицы $R_{1}, R_{2}$ симметричны, т.е. $R_{1}=$ $R_{1}^{t}$ и $R_{2}=R_{2}^{t}$. Тогда легко проверить [14], что матрица $U(x, t)$ также симметрична: $U^{t}(x, t)=U(x, t)$, и потенциал является неособым для любого $t$. Изменение поляризаций солитонов при взаимодействии задается формулами

$$
P_{1}^{-}=\frac{p_{1} \otimes p_{1}}{\left|p_{1}\right|^{2}} \rightarrow P_{1}^{+}=\frac{\xi_{1} \otimes \xi_{1}}{\left|\xi_{1}\right|^{2}}, \quad P_{2}^{-}=\frac{\xi_{2} \otimes \xi_{2}}{\left|\xi_{2}\right|^{2}} \rightarrow P_{2}^{+}=\frac{p_{2} \otimes p_{2}}{\left|p_{2}\right|^{2}}
$$

где

$$
\xi_{1}=p_{2}^{2} p_{1}-\frac{2 \lambda_{1}\left(p_{1}, p_{2}\right) p_{2}}{\lambda_{1}+\lambda_{2}}, \quad \xi_{2}=p_{1}^{2} p_{2}-\frac{2 \lambda_{2}\left(p_{1}, p_{2}\right) p_{1}}{\lambda_{1}+\lambda_{2}}
$$

а сдвиги фаз

$$
\Delta_{1}=\frac{1}{2 \lambda_{1}} \ln \left(\sin ^{2} \beta+\left(\frac{\lambda_{1}-\lambda_{2}}{\lambda_{1}+\lambda_{2}}\right)^{2} \cos ^{2} \beta\right)<0
$$

и

$$
\Delta_{2}=-\frac{1}{2 \lambda_{2}} \ln \left(\sin ^{2} \beta+\left(\frac{\lambda_{1}-\lambda_{2}}{\lambda_{1}+\lambda_{2}}\right)^{2} \cos ^{2} \beta\right)>0,
$$

где $\beta=\angle\left(p_{1}, p_{2}\right)$, т.е., как и в скалярном случае, медленный солитон сдвигается назад, а быстрый - вперед. Однако в противоположность скалярному случаю [2] солитоны с близкими скоростями не взаимодействуют интенсивно. Если $\lambda_{2} \rightarrow \lambda_{1}=\lambda$, то

$$
\Delta_{1} \rightarrow \frac{1}{2 \lambda} \ln \sin ^{2} \beta, \quad \Delta_{2} \rightarrow-\frac{1}{2 \lambda} \ln \sin ^{2} \beta
$$

и стремятся к бесконечности, если только $\beta=0$, когда матричный случай сводится к скалярному. Отметим также, что в пределе $\lambda_{2} \rightarrow \lambda_{1}=\lambda$ решение (15) стремится к решению (13) с $R=R_{1}+R_{2}$, описываюшему пару невзаимодействующих параллельных солитонов. Формула (17) означает, что предельные переходы $\lambda_{2} \rightarrow \lambda_{1}$ и $t \rightarrow \pm \infty$ не коммутируют, что, впрочем, имеет место и в скалярном случае. 
Общий $N$-солитонный случай соответствует безотражательному оператору Шредингера с $N$ дискретными собственными значениями $-\lambda_{N}^{2}<\cdots<-\lambda_{1}^{2}$. Предположим, что соответствующие спектральные матрицы имеют вид

$$
R_{j}=r_{j} P_{j}, \quad P_{j}=\frac{p_{j} \otimes q_{j}}{\left(p_{j}, q_{j}\right)}, \quad j=1, \ldots, N
$$

где $p_{j}, q_{j}-d$-векторы такие, что скалярные произведения $\left(p_{j}, q_{j}\right) \neq 0$. Аналогично случаю $N=2$ можно показать, что решение (12) при $t \rightarrow \pm \infty$ распадается в сумму $N$ солитонов с амплитудами $R_{j}^{ \pm}$, фазами $\phi_{j}^{ \pm}$и скоростями $4 \lambda_{j}^{2}$. Заметим, далее, что в работе [15] была построена иерархия потоков, коммутирующих с потоком матричного уравнения Кд $\Phi$, и доказано, что они допускают представление Лакса. Действуя одним из высших потоков уравнения КдФ, мы, очевидно, можем добиться того, что при взаимодействии $i$-го и $j$-го солитонов остальные солитоны не будут влиять на их взаимодействие. Таким образом, мы приходим к следующей теореме.

ТЕОРема 2. При взаимодействии $N$ солитонов матричного уравнения КдФ полныи сдвиг фазы $\Delta_{j}=\phi_{j}^{+}-\phi_{j}^{-}$и полное изменение амплитуды $R_{j}^{-} \rightarrow R_{j}^{+}$каждого солитона определяются его попарным взаимодействием с остальными солитонами и не зависят от того, в каком порядке оно происходит. Многочастичнье эффекты при солитонном взаимодействии отсутствуют.

\section{3. МАТРИЧНОЕ ПРЕОБРАЗОВАНИЕ ДАРБУ И СОЛИТОНЫ}

Хорошо известно, что солитонные решения скалярного уравнения КдФ могут быть получены с помощью преобразований Дарбу (см., например, [3]). В матричном случае мы можем использовать общую схему работы [12] для получения решений матричного уравнения КдФ в виде

$$
U=-2\left(Y_{n} W_{n}^{-1}\right)^{\prime}
$$

где

$$
\begin{gathered}
W=W\left(\Psi_{1}, \ldots, \Psi_{n}\right)=\left(\begin{array}{ccc}
\Psi_{1} & \ldots & \Psi_{n} \\
\vdots & \ddots & \vdots \\
\Psi_{1}^{(n-2)} & \ldots & \Psi_{n}^{(n-2)} \\
\Psi_{1}^{(n-1)} & \ldots & \Psi_{n}^{(n-1)}
\end{array}\right), \\
Y=Y\left(\Psi_{1}, \ldots, \Psi_{n}\right)=\left(\begin{array}{ccc}
\Psi_{1} & \ldots & \Psi_{n} \\
\vdots & \ddots & \vdots \\
\Psi_{1}^{(n-2)} & \ldots & \Psi_{n}^{(n-2)} \\
\Psi_{1}^{(n)} & \ldots & \Psi_{n}^{(n)}
\end{array}\right),
\end{gathered}
$$

$Y_{n}=|Y|_{n n}, W_{n}=|W|_{n n}$, а матричные функции $\Psi_{1}, \ldots, \Psi_{n}$ являются столбцами $(d \times$ $n d$ )-матрищы $\Phi$, удовлетворяющей уравнению

$$
\Phi^{\prime \prime}=\Phi C,
$$


причем постоянная $(n d \times n d)$-матрица $C$ имеет диагональный вид

$$
C=\left(\begin{array}{cccc}
\lambda_{1}^{2} I_{1} & 0 & \ldots & 0 \\
0 & \lambda_{2}^{2} I_{2} & \ldots & 0 \\
0 & \ldots & \ddots & 0 \\
0 & \ldots & 0 & \lambda_{N}^{2} I_{N}
\end{array}\right)
$$

где $I_{k}$ - единичные матрицы размера $\left(n_{k} \times n_{k}\right)$ и $\sum_{k=1}^{N} n_{k}=n d$. Оператор $L=-D^{2}+U$ с потенциалом (18) связан матричным преобразованием Дарбу с $L_{0}=-D^{2}$, т.е. сушествует матричный дифференциальньй оператор $A$ порядка $n$, сплетающий $L=-D^{2}+U$ и $L_{0}=-D^{2}($ см. [12]):

$$
L A=A L_{0}
$$

Зависимость от времени задается уравнением (см. [3], [13]) $\Phi_{t}=-4 D^{3} \Phi$, т.е. функции $\Psi_{j}$ имеют вид

$$
\Psi_{j}=P_{j} e^{\Lambda_{j} x-4 \Lambda_{j}^{3} t}+Q_{j} e^{-\Lambda_{j} x+4 \Lambda_{j}^{3} t},
$$

где $P_{j}, Q_{j}$ - постоянные матрицы, а $\Lambda_{j}, j=1, \ldots, n$, являются положительно определенными $(d \times d)$-матрищами, которые получаются из представления $C$ в виде

$$
C=\left(\begin{array}{cccc}
\Lambda_{1}^{2} & 0 & \ldots & 0 \\
0 & \Lambda_{2}^{2} & \ldots & 0 \\
0 & \ldots & \ddots & 0 \\
0 & \ldots & 0 & \Lambda_{n}^{2}
\end{array}\right) \text {. }
$$

Как недостаток метода Дарбу следует упомянуть сложность контроля за симметричностью соответствуюшего решения.

Рассмотрим теперь простейший нетривиальный случай, когда $A$ является оператором первого порядка. Тогда $C=\Lambda^{2}, \Lambda=\operatorname{diag}\left(\lambda_{1} I_{1}, \ldots, \lambda_{N} I_{N}\right)$ и $\sum_{k=1}^{N} n_{k}=d$. Уравнение (19) может быть записано в виде $\Psi^{\prime \prime}=\Psi \Lambda^{2}$. Тогда

$$
\Psi=P e^{\Lambda x}+Q e^{-\Lambda x}
$$

где $P$ и $Q-$ произвольные матришы (мы будем предполагать, что они невырожденны) и

$$
U=-2\left(\Psi^{\prime} \Psi^{-1}\right)^{\prime}
$$

Для сравнения решения (21) с многосолитонными решениями, обсуждавшимися ранее, вычислим спектральные данные $\left(\lambda_{j}, R_{j}\right)$ через параметры преобразований Дарбу $(\Lambda, P, Q)$. Заметим, что

$$
\begin{gathered}
\lim _{x \rightarrow+\infty} \Psi^{\prime} \Psi^{-1}=\lim _{x \rightarrow+\infty}\left(\Psi^{\prime} e^{-\Lambda x}\right)\left(\Psi e^{-\Lambda x}\right)^{-1}=P \Lambda P^{-1}, \\
\lim _{x \rightarrow-\infty} \Psi^{\prime} \Psi^{-1}=-Q \Lambda Q^{-1} .
\end{gathered}
$$


Используя квазидетерминантную формулу (см. [12], [13]) для оператора $A$, легко проверить, что

$$
\Phi_{1}(x, k)=\left|\begin{array}{cc}
\Psi & I e^{i k x} \\
\Psi^{\prime} & i k I e^{i k x}
\end{array}\right|_{22}=\left(i k I-\Psi^{\prime} \Psi^{-1}\right) e^{i k x}
$$

И

$$
\Phi_{2}(x, k)=\left|\begin{array}{cc}
\Psi & I e^{-i k x} \\
\Psi^{\prime} & -i k I e^{-i k x}
\end{array}\right|_{22}=-\left(i k I+\Psi^{\prime} \Psi^{-1}\right) e^{-i k x}
$$

являются решениями уравнения

$$
L \Phi=k^{2} \Phi, \quad L=-D^{2}+U(x),
$$

а из формул (22) заключаем, что решения Йоста уравнения (23) имеют вид

$$
\begin{aligned}
& F(x, k)=\Phi_{1}(x, k) P(i k I-\Lambda)^{-1} P^{-1} e^{i k x} \\
& G(x, k)=\Phi_{2}(x, k) Q(i k I-\Lambda)^{-1} Q^{-1} e^{-i k x} .
\end{aligned}
$$

Так как $\Phi_{1}(x, k)=\Phi_{2}(x,-k)$ (потенциал $(21)$, очевидно, безотражательньй), то

$$
A(k)=F^{-1}(x,-k) G(x, k)=P(i k I+\Lambda) P^{-1} Q(i k I-\Lambda)^{-1} Q^{-1}
$$

и

$$
\operatorname{det} A(k)=\frac{\operatorname{det}(i k I+\Lambda)}{\operatorname{det}(i k I-\Lambda)} .
$$

Таким образом, дискретный спектр оператора $L$ с потенциалом (21) состоит из точек $-\lambda_{j}^{2}$, где $\lambda_{j}$ - собственные значения матрицы $\Lambda$, и (21) является $N$-солитонным решением матричного уравнения $\mathrm{K}$ д $\Phi$.

Спектральные данные $R_{j}$ определяются условиями (6),

$$
\left(-\lambda_{j} I+\Psi^{\prime} \Psi^{-1}\right) e^{\lambda_{j} x} P\left(\operatorname{Res}_{k=i \lambda_{j}}(i k I+\Lambda)^{-1}\right) P^{-1}=F\left(x, k_{j}\right) R_{j} .
$$

Так как $F\left(x, i \lambda_{j}\right)=(I+o(1)) e^{-\lambda_{j} x}$, когда $x \rightarrow+\infty$, то, рассматривая последнее выражение в пределе $x \rightarrow+\infty$, мы получаем

$$
R_{j}=\lim _{x \rightarrow+\infty}\left[\left(-\lambda_{j} I+\Psi^{\prime} \Psi^{-1}\right) P P_{j} P^{-1} e^{2 \lambda_{j} x}\right]
$$

где $P_{j}=\operatorname{Res}_{k=i \lambda_{j}}(i k I+\Lambda)^{-1}=\operatorname{diag}\left(0, \ldots, 0, I_{j}, 0, \ldots, 0\right)$ суть проекторы на собственные подпространства, соответствуюшие собственным значениям $\lambda_{j}$ матрицы $\Lambda$.

ПредложениЕ 2. Спектральные данные $R_{j}$ оператора Шредингера L с потенциалом (21) выражаются через параметры преобразований Дарбу формулами

$$
R_{j}=\operatorname{Pf}\left(\lambda_{j}\right) P^{-1} Q P_{j} P^{-1},
$$

əде $f(\lambda)=\lambda I+\Lambda$. 
ДОкАЗАТЕЛЬСтво. Мы можем переписать (24) в виде

$$
R_{j}=\lim _{x \rightarrow+\infty}\left[\left(-\lambda_{j} \Psi-\Psi^{\prime}\right)\left(P^{-1} \Psi\right)^{-1} P_{j} P^{-1} e^{2 \lambda_{j} x}\right] .
$$

Тогда

$$
\left(P^{-1} \Psi\right)^{-1}=e^{-\Lambda x}\left(I+P^{-1} Q e^{-2 \Lambda x}\right)^{-1}=e^{-\Lambda x}\left(I-P^{-1} Q e^{-2 \Lambda x}+\cdots\right)
$$

и прямым вычислением, используюшим коммутативность $P_{j}, j=1, \ldots, N$, мы приходим к доказательству предложения.

Предложение 2 можно использовать для нахождения условий на параметры преобразования Дарбу, гарантируюших симметричность потенциала. Действительно, потенциал (21) симметричен тогда и только тогда, когда симметричны спектральные матришы $R_{j}, j=1, \ldots, N: R_{j}^{t}=R_{j}$, что может быть записано в виде

$$
P f\left(\lambda_{j}\right) P^{-1} Q P_{j} P^{-1}=\left(P^{t}\right)^{-1} P_{j} Q^{t}\left(P^{-1}\right)^{t} f\left(\lambda_{j}\right) P^{t} .
$$

Легко проверить, что

$$
Q=\sum_{j=1}^{N} P f^{-1}\left(\lambda_{j}\right)\left(P^{t} P\right)^{-1} P_{j}
$$

являются решениями (25). Используя предложение 2 , мы получаем следуюшее

ПрЕДЛОЖЕНИЕ 3. Потенииал (21) симметричен, если матрицы $Q$ определяются через $P$ по формулам (26). Тогда

$$
R_{j}=\left(P^{t}\right)^{-1} P_{j} P^{-1} .
$$

Пример. Рассмотрим случай $d=2, \quad \Lambda=\operatorname{diag}\left(\lambda_{1}, \lambda_{2}\right), \quad \lambda_{1} \neq \lambda_{2}$. Потенциал (21) удовлетворяет матричному уравнению $\mathrm{K} д \Phi$, если

$$
\Psi=P(t) e^{\Lambda x}+Q(t) e^{-\Lambda x}, \quad P(t)=P e^{-4 \Lambda^{3} t}, \quad Q(t)=Q e^{4 \Lambda^{3} t}
$$

и

$$
P=\left(\begin{array}{ll}
p_{11} & p_{12} \\
p_{21} & p_{22}
\end{array}\right), \quad Q=\left(\begin{array}{ll}
q_{11} & q_{12} \\
q_{21} & q_{22}
\end{array}\right) .
$$

Предположим, что $\operatorname{det} P \neq 0, \operatorname{det} Q \neq 0$, и введем

$$
\widehat{P}=\left(\begin{array}{ll}
p_{11} & q_{12} \\
p_{21} & q_{22}
\end{array}\right), \quad \widehat{Q}=\left(\begin{array}{ll}
q_{11} & p_{12} \\
q_{21} & p_{22}
\end{array}\right) .
$$

Легко проверить, используя предложение 3 и пример 2 раздела 2 , что сдвиги фаз могут быть выражены через параметры матричных преобразований Дарбу формулами

$$
\Delta_{1}=\frac{1}{2 \lambda_{1}} \ln \frac{\operatorname{det} \widehat{P} \operatorname{det} \widehat{Q}}{\operatorname{det} P \operatorname{det} Q}, \quad \Delta_{2}=\frac{1}{2 \lambda_{2}} \ln \frac{\operatorname{det} P \operatorname{det} Q}{\operatorname{det} \widehat{P} \operatorname{det} \widehat{Q}} .
$$


Далее,

$$
\begin{aligned}
\operatorname{det} \Psi= & e^{\left(\lambda_{1}+\lambda_{2}\right) x-4\left(\lambda_{1}^{3}+\lambda_{2}^{3}\right) t} \operatorname{det} P+e^{\left(\lambda_{1}-\lambda_{2}\right) x-4\left(\lambda_{1}^{3}-\lambda_{2}^{3}\right) t} \operatorname{det} \widehat{P}+ \\
& +e^{\left(\lambda_{2}-\lambda_{1}\right) x+4\left(\lambda_{1}^{3}-\lambda_{2}^{3}\right) t} \operatorname{det} \widehat{Q}+e^{-\left(\lambda_{1}+\lambda_{2}\right) x+4\left(\lambda_{1}^{3}+\lambda_{2}^{3}\right) t} \operatorname{det} Q,
\end{aligned}
$$

и потенциал $U(x, t)$ регулярен, если $\operatorname{det} \Psi \neq 0$ для любого $x$, что выполнено при

$$
\operatorname{det} P>0, \quad \operatorname{det} \widehat{P}>0, \quad \operatorname{det} Q>0, \quad \operatorname{det} \widehat{Q}>0 .
$$

Введем векторы $e_{1}=\left(p_{11}, p_{21}\right), e_{2}=\left(p_{12}, p_{22}\right), e_{3}=\left(q_{11}, q_{21}\right), e_{4}=\left(q_{12}, q_{22}\right)$ и углы между ними $\alpha=\angle\left(e_{1}, e_{3}\right), \beta=\angle\left(e_{1}, e_{2}\right), \gamma=\angle\left(e_{1}, e_{4}\right)$. Тогда условия регулярности (28) эквивалентны одному из условий $0<\alpha<\beta<\gamma<\pi$ или $0<\alpha<\gamma<\beta<\pi$ и

$$
\frac{\operatorname{det} \widehat{P} \operatorname{det} \widehat{Q}}{\operatorname{det} P \operatorname{det} Q}=\frac{\sin (\beta-\alpha) \sin \gamma}{\sin \beta \sin (\gamma-\alpha)}=\frac{\cos \alpha-\sin \alpha \operatorname{ctg} \beta}{\cos \alpha-\sin \alpha \operatorname{ctg} \gamma} \text {. }
$$

Из этой формулы видно, что если $\beta<\gamma$, то $\Delta_{1}<0\left(\Delta_{2}>0\right)$, а если $\beta>\gamma$, то $\Delta_{1}>0$ $\left(\Delta_{2}<0\right)$.

Благодарности. Автор хотел бы выразить свою искреннюю благодарность профессору А. П. Веселову за постановку задачи, частично решенной в этой работе, и многочисленные дискуссии. Работа была поддержана грантами университета Лафборо и INTAS 96-1770.

\section{Список литературы}

[1] C. Gardner, J. Green, M. Kruskal, R. Miura. Phys. Rev. Lett. 1967. V. 19. P. 1095.

[2] В.Е. Захаров, С.В. Манаков, С. П. Новиков, Л. П. Питаевский. Теория солитонов: Метод обратной задачи. М.: Наука, 1980.

[3] V. B. Matveev, M. A. Salle. Darboux Transformations and Solitons. Berlin: Springer, 1991.

[4] P. D. Lax. Commun. Pure and Appl. Math. 1968. V. 21. P. 467.

[5] M. Wadati, T. Kamijo. Prog. Theor. Physics. 1974. V. 52. P. 397.

[6] F. Calogero, A. Degasperis. Nuovo Cimento. 1977. V. 39B. № 1. P. 1.

[7] A. Degasperis. Solitons, boomerons, trappons. In: Nonlinear Evolutional Equations Solvable by Spectral Transform. Research Notes in Math. V. 26. Ed. F. Calogero. London: Pitman Publishing, 1978. P. 97.

[8] L. Martinez Alonso, E. Olmedilla. J. Math. Phys. 1982. V. 23. № 11. P. 2116.

[9] E. Olmedilla. Inverse Problem. 1985. V. 1. P. 219.

[10] И. М. Гельфанд, В. С. Ретах. Функц. анализ и его прилож. 1991. Т. 25. № 2. С. 13.

[11] В. М. Гончаренко. УМН. 2000. Т. 55. № 5. С. 175-176.

[12] V. M. Goncharenko, A. P. Veselov. J. Phys. A. 1998. V. 31. P. 5315.

[13] P. Etingof, I. Gelfand, V. Retakh. Math. Res. Lett. 1997. V. 4. № 2-3. P. 413; q-alg/9701008.

[14] З. С. Агранович, В. А. Марченко. Обратная задача теории рассеяния. Харьков: Изд-во Харьковского ун-та, 1960.

[15] E. Olmedilla, L. Martinez Alonso, F. Guil. Nuovo Cimento B. 1981. V. 61. № 1. P. 49. 